da sie sich doch ziemlich weitgehend bewährt hatte. Mit Hilfe des Boydschen Schichtkriteriums konnte zudem nachgewiesen werden, daß die Verhältnisse unter recht weitgehenden Bedingungen sogar günstiger liegen als für den Fall eines nur Elektronen enthaltenden Plasmas, nämlich solange nicht $\mathrm{T}_{+}<\mathrm{T}_{-}$und zugleich $n_{-} / n_{e}$ nicht zu groß sind. Außerdem wurden für alle drei Trägerarten (Elektronen, negative und positive Ionen) je eine Maxwell-Verteilung der Geschwindigkeiten angenommen und den Trägern demgemä $ß$ die Temperaturen $T_{e}, T_{-}$und $T_{+}$zugeordnet.

Die Theorie konnte bis zu unmittelbar auswertbaren Endformeln entwickelt werden für die unendliche und die quadratische bzw. kreisförmige begrenzte Plansonde sowie für die Zylinder- und die Kugelsonde, umfaßt also alle meistens benutzten Sondenformen. Eine eingehendere Darstellung ist ohne sehr viele Formeln und die Erklärung aller einzuführenden Hilfsgrößen bzw. Bezeichnungen nicht möglich. Grundsätzlich kommt zu den nun entsprechend verallgemeinerten Ausgangsgleichungen, soweit es sich um die kinematischen Gleichungen handelt (Orbitalbewegung), einfach ein additives Glied hinzu; für den Fall der Raumladungsbegrenzung muß nun die negative Raumladungsdichte in die Poissonsche Gleichung eingeführt werden und dies hat ernstlichere Komplikationen zur Folge. Aber auch sie konnte mit dem durch die negativen Ionen bedingten Zusatzglied integriert werden, und zwar nicht nur unter Vernachlässigung der Anfangsgeschwindigkeiten der Träger.

Wie bereits erwähnt, ist für die genannten Sondentypen das Endergebnis die Formulierung quantitativ gültiger Anweisungen dafür, wie man vorgehen muß, um aus der Sondencharakteristik die Plasma-Kenngrößen zu bestimmen.

\title{
Das Energiespektrum hoher Auflösung von Kathodenstrahlen aus der Gasentladung
}

\author{
Von G. Möllenstedt und H. Düke R
}

\begin{abstract}
Aus den Süddeutschen Laboratorien Mosbach und dem Physikalischen Institut der Universität Tübingen

Z. Naturforschg. 8a, 79-90 [1953]; eingegangen am 25. Oktober 1952)
\end{abstract}

Herrn Professor Dr. E. Fues zum 60. Geburtstag gewidmet

1. Mittels magnetischer Umlenkung wurde die bereits von Gerthsen und Fauldraht gefundene chromatische Zusammensetzung von Elektronen aus einer Gasentladung bestätigt: Die Zahl der Elektronen, deren Energie zwischen $0 \%$ und $99 \%$ der Energie liegt, die der Kathodenspannung eñtspricht, beträgt weniger als $1 \%$ der Gesamtintensität. Als Entladungsrohr wurde wegen guter Strom- und Spannungskonstanz. die Indunische Bauart benutzt.

2. Mittels des hochauflösenden elektrostatischen Geschwindigkeits-Analysators konnten die folgenden Ergebnisse erzielt werden.

a) Das hochaufgelöste Energiespektrum von $40-\mathrm{kV}$-Elektronen aus der Gasentladung besteht im wesentlichen aus einer asymmetrischen Linie mit einer Halbwertsbreite von $2 \mathrm{eV}$. Die steile Flanke befindet sich auf der energieärmeren Seite. Die spektrale Verteilung ist schmaler als die, die Baerwald mittels Gegenfeldmethode gefunden hat. Damit bestätigt sich die von Induni aus dem hohen Auflösungsvermögen des Schweizer Elektronenmikroskops vorausgesagte Monochromasie der Elektronen aus der Gasentladungsquelle. Neben dieser Linie zeigt sich in einem Abstand von $13 \mathrm{eV}$ nach der Seite der Geschwindigkeitsverluste eine sch wache aber scharfe Kante, die der ersten Anregungsmöglichkeit des Entladungsgases entspricht. Daran schließt sich ein kontinuierlicher Untergrund an, dessen Intensität 2 bis 3\% der Gesamtintensität beträgt.

b) Die spektrale Energieverteilung der Hauptlinie ändert sich nicht bei Variation des Kathodenmaterials (V2A, Al, Pt), des Entladungsgases (Luft, Wasserstoff, Stickstoff) oder bei Änderung der Hochspannung von 25 bis $45 \mathrm{kV}$.

c) Die spektrale Breite der Hauptlinie ändert sich auch nicht, wenn statt einer ,,materiellen" Kathode eine durchbohrte nach Hailer verwendet wird. Ebenso tritt keine Änderung auf, wenn die Ionen aus einem gesonderten Entladungsraum auf die im besseren Vakuum $\left(10^{-3}\right.$ bis $\left.10^{-4} \mathrm{~mm} \mathrm{Hg}\right)$ befindliche Kathode geschossen werden und dort die Elektronen zur Auslösung kommen.

d) Es wird vermutet, daß die Ursache für die Asymmetrie der Hauptlinie in der nicht einheitlichen Geschwindigkeit der auf die Kathode prallenden Ionen liegt. 
$\mathrm{D}$ urch die stetige Weiterentwicklung der dynamischen Theorie der Elektronen-Interferenzen durch Fues wird uns ein immer besserer Einblick in die feinsten Züge der Ausbreitung von Elektronenwellen im Kristallgitter ermöglicht. Auch die Experimentatoren verfeinern und verbessern sowohl auf dem Gebiet der Elektronen-Interferenzen wie auch in der Elektronenmikroskopie ihre Verfahren. Ob für Interferenz- und Abbildungs-Versuche die Elektronenstrahlen einer Gasentladungsquelle oder Glühkathode entnommen werden, ist keineswegs gleichgültig. Zum Beispiel ist die Kohärenzlänge von Elektronenstrahlen $\lambda^{2} / \Delta \lambda$ eine Funktion der chromatischen Zusammensetzung des Elektronenstrahls. Die im folgenden mit dem hochauflösenden elektrostatischen GeschwindigkeitsAnalysator ausgeführte Analyse von Elektronenstrahlen aus einer Gasentladung soll uns über die spektrale Breite $\Delta \lambda$ Auskunft geben.

Einen ersten Hinweis in dieser Richtung gibt uns das Elektronenmikroskop der Firma Trüb, Täu ber \& Co, Zürich. Es verwendet als einziges Gerät

${ }^{1}$ B. v. Borries u. E. Ruska.

${ }^{2}$ G. Induni, Helv. physica Acta 20, 463 [1947].

${ }^{3}$ Der Direktion der Firma Trüb. Täuber \& Co., Zü - von den auf dem Weltmarkt befindlichen Typen statt der sonst üblichen Glühemission die Gasentladung. Die Gründe, weswegen man in den Anfängen der Elektronenmikroskopie in Deutschland ${ }^{1}$ von der Gasentladung zur Glühemission überging, waren, neben der damals nicht voll ausreichenden Intensität, die Abhängigkeit der Strahlspannung von der Stromstärke und ganz besonders aber das Mißtrauen gegenüber der Monochromasie der Strahlung. Trotz dieser Sachlage hat Induni ${ }^{2}$ sein Gerät mit einer neuartigen Gasentladungskathode versehen und erzielt damit ausgezeichnete Bilder. Die praktisch erreichte Auflösungsgrenze des mit einem elektrostatischen Objektiv versehenen Mikroskops liegt bei 20-30 AE. Das in Abb. 1 wiedergegebene Bild $^{3}$ wurde mit Elektronen aus einer Gasentladungsquelle Indunischer Bauart bei 11000 -facher elektronenoptischer Vergrößerung aufgenommen. Die Gesamtvergrößerung beträgt 25000. Es stellt Wolframoxyd dar, das zur besseren Erkennung der räumlichen Anordnung der Teilchen mit Gold schräg beschattet wurde. Die Granulation des Untergrun-

rich, sind die Verfasser für die freundliche Überlassung der in Abb. 1 wiedergegebenen Aufnahme zu Dank verpflichtet.

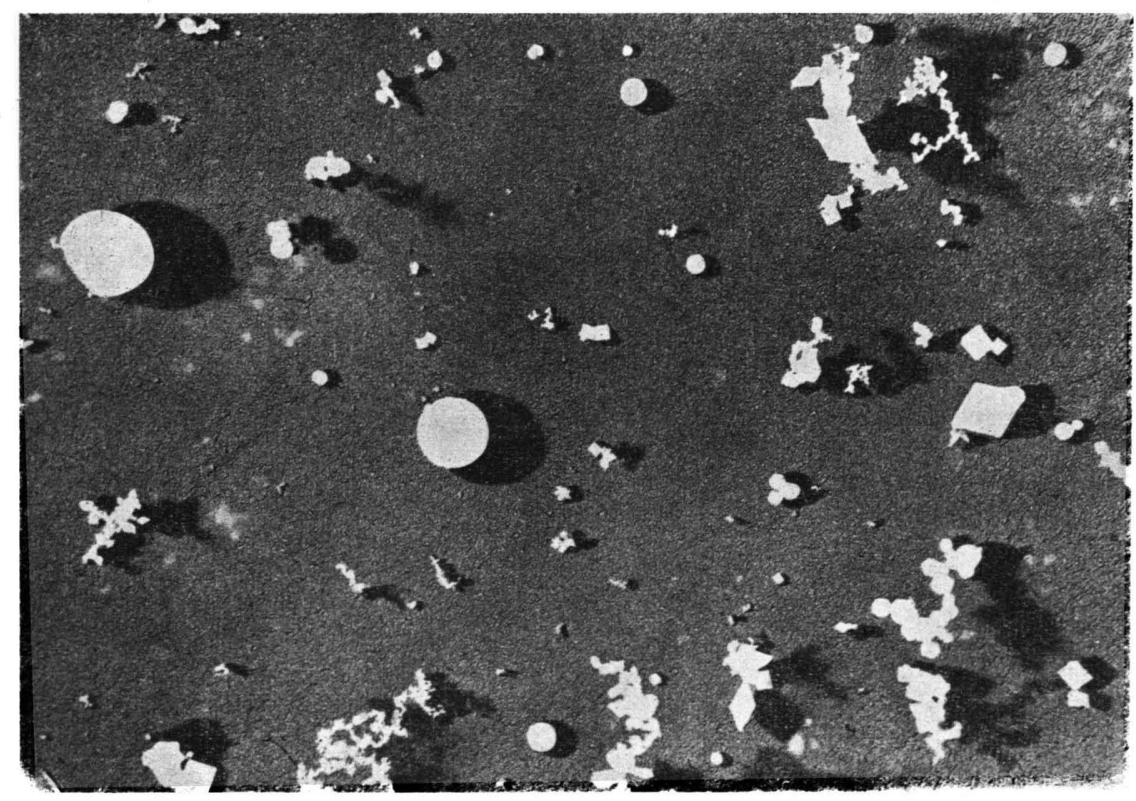

Abb. 1. Mit Elektronen aus einer Gasentladungsquelle Indunischer Bauart aufgenommen. Objekt: Wolframoxyd, mit Gold schräg beschattet. Elektronenopt. Vergrößerung: 11000 . Gesamtvergr. :25000. (Gerät Trüb, Täuber \& Co., Zürich). 
des enthält mit guter Zeichenschärfe wiedergegebene Goldkriställchen. Aus der Formel für den chromatischen Streukreis

$$
\varrho \mathrm{Chr}, \mathrm{Obj}=p \alpha f \frac{\Delta U}{U},
$$

$p=\tilde{5}$ :chromatische Linsenkonstante für elektrostatisches Objektiv, $\alpha=3 \cdot 10^{-3}$ : Apertur in Bogenmaß, $f=5 \mathrm{~mm}$ : Brennweite des Objektivs, $U=$ $50 \mathrm{kV}$ : Kathodenspannung, $e \Delta U=$ Spektrale Streubreite der Energie der Elektronen im Strahl, schloß Induni, daß solch gute Zeichenschärfe nur möglich sei, wenn die Energieverteilung der Elektronen aus der Entladung bis auf etwa $\mathrm{l} \mathrm{eV}$ gegeben ist.

Da die hohe Monochromasie der GasentladungsElektronen bisher unbekannt und auch nicht ohne weiteres $\mathrm{zu}$ erwarten ist, lohnt es sich, die Geschwindigkeitsverteilung mit dem hochauflösenden, elektrostatischen Geschwindigkeits-Analysator unmittelbar festzustellen.

1. Bisherige Kenntnis über die chromatische Zusammensetzung von Elektronen aus der Gasentladung

\section{Messungen von Fauldraht ${ }^{4}$}

Fauldraht bestimmte auf Anregung von Gerthsen, in seiner Dissertation ,Über die Energieverteilung von Kathodenstrahlen aus einer Gasentladung "* die Geschwindigkeitsverteilung von Elektronen aus einer bei 8 bis $25 \mathrm{kV}$ brennenden Gasentladung. Die Analyse wurde durch elektrostatische Umlenkung eines unter $45^{0}$ in einen Plattenkondensator eingeschlossenen Elektronenbündels erreicht (Abb. 2). Dabei stellte sich heraus, daß die Zahl der Elektronen, deren Energie zwischen 0 und $85 \%$ der Gesamtenergie entsprechend der Kathodenspannung

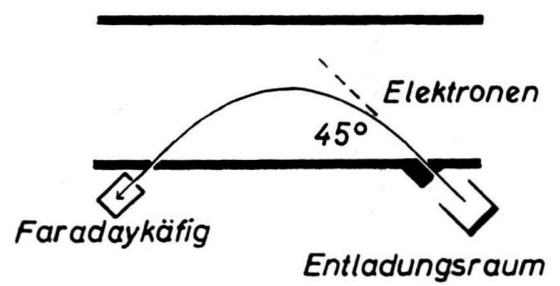

Abb. 2. Von Gerthsen und Fauldraht benutzte Versuchsanordnung zur Geschwindigkeits-Analyse von Kathodenstrahlen aus der Gasentladung. Die obere Flatte ist die negativ aufgeladene.

${ }^{4}$ Herr Professor Dr. Chr. Gerthsen, Direktor des Physikalischen Instituts der Technischen Hochschule Karlsruhe, gewährte uns in dankenswerter Weise Ein- liegt, kleiner als $0,01 \%$, zwischen 85 und $95 \%$ kleiner als $0,02 \%$ und zwischen 95 und $99 \%$ kleiner als $0,2 \%$ der maximalen Intensität ist. Die Energieverteilung der Elektronen von der Energie zwischen 99 und $100 \%$ der Kathodenspannung konnte Fauldraht nicht feststellen, da sein Analysator nicht das ausreichende Trennvermögen besaß.

\section{Beschreibung der im folgenden benutz. ten Entladungsquelle}

Für die im folgenden beschriebene Geschwindigkeits-Analyse von Kathodenstrahlen wurde unter $\mathrm{Zu}$ hilfenahme der Bauelemente der elektrostatischen Elektronenmikroskopie eine Quelle mit den von Induni angegebenen charakteristischen Abmessungen benutzt. Für konstantes Brennen der Entladung ist wichtig, daß die von Induni empirisch gefundene $\mathrm{Be}$ ziehung $d \leqq \lambda_{\mathrm{m}} \leqq D$ eingehalten wird; es bedeuten

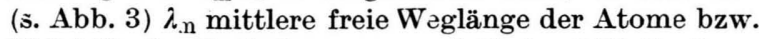
Moleküle des Entladungsgases, $d$ Abstand Kathode Anode, $D$ Durchmesser der Anodenblende. Da die freie Weglänge in Luft bei einem Druck von $10^{-2} \mathrm{~mm} \mathrm{Hg}$ einige Millimeter beträgt, so ist nach dieser Beziehung im Gegensatz zu den sonst üblichen Entladungsquellen der Abstand Kathode - Anode sehr eng gehalten.

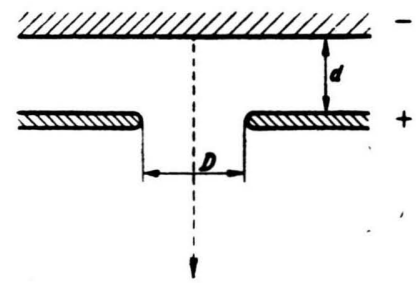

Abb. 3. Zur Dimensionierung der Gasentladungskathode nach Induni.

Die Entladung kann, wie es durch den Hittorfschen Umwegversuch so deutlich demonstriert wird, nur in der Öffnung $D$ brennen. Abb. 4 stellt den Schnitt durch die hier benutzte technische Ausführung der Kathode dar. Die Hochspannung von $40 \mathrm{kV}$ wird an die als Rundstange ausgeführte Aluminiumkathode gelegt. Für die Hochspannungszuführung dient die übliche für das elektrostatische Übermikroskop benutzte Durchführung aus Porzellan. Die Anode besteht aus einem auf Erdpotential befindlichen Eisenrohr mit der Bohrung $D=5 \mathrm{~mm}$ (Blende 1). Die Öffnung in Blende 2 besitzt einen Durchmesser von nur $0,1 \mathrm{~mm}$ und hat die Aufgabe, einen Feinstrahl auszublenden. Der Gaseinlaß geschieht über das äußerst fein regulierbare Ventil nach $\mathrm{Ewald}^{5}$ gegen Atmosphärendruck. Es wird durch ein seitlich in den Entladungsraum angebrachtes Loch abgepumpt. Mittels

blick in die bisher noch nicht veröffentlichte Dissertation von L. Fauldraht. Dissertation Berlin $1942 / 43$.

${ }^{5}$ H. Ewald, Z. Naturforschg. 5a, 230 [1950]. 


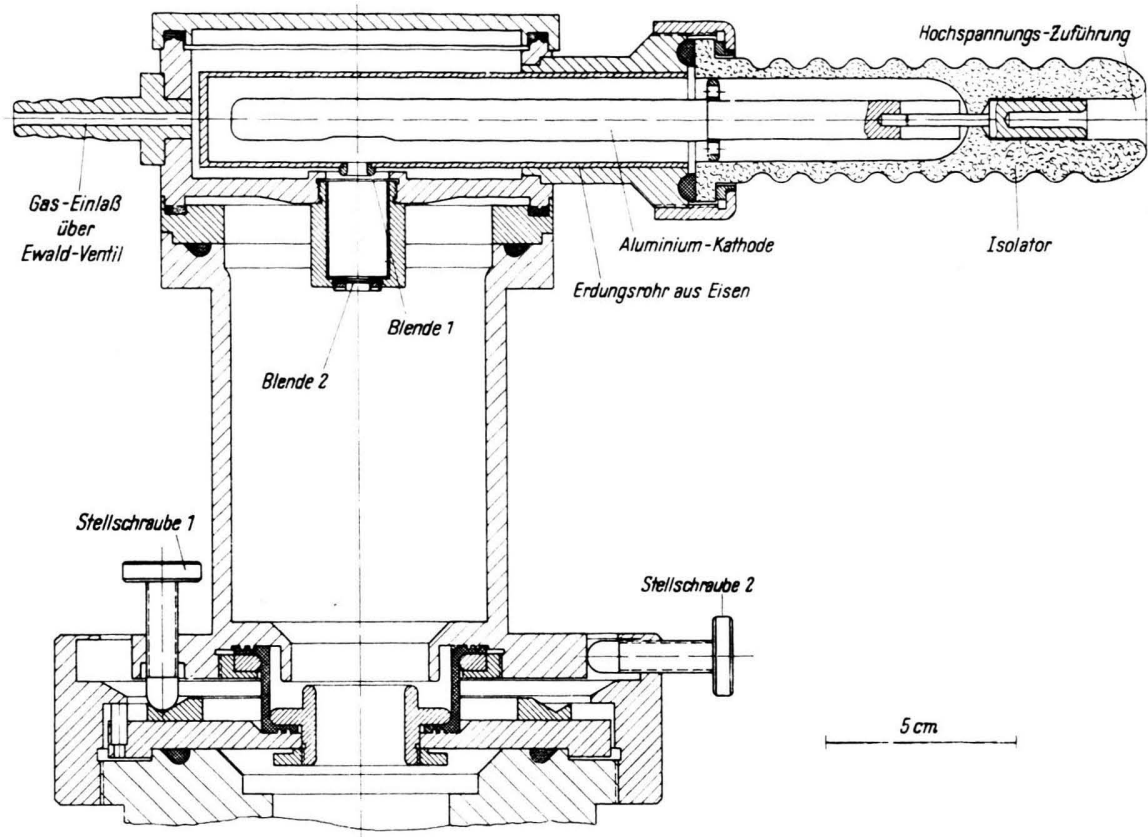

Abb. 4. Schnitt durch die technische Ausführung der Gasentladungskathode nach Induni.

sechs Stellschrauben, von denen in der Zeichnung 1 und $2 \mathrm{zu}$ sehen sind, ist die Elektronenquelle sowohl kippbar als auch nach der Seite verschiebbar.

\section{Geschwindigkeits-Analyse von Elektro- nen im Magnetfeld der Erde}

Schon das schwache Magnetfeld der Erde demonstriert uns die chromatische Zusammensetzung der aus der Gasentladung stammenden Elektronen. Der fein ausgeblendete, aus der oben beschriebenen Gasentladungskathode kommende Elektronenstrahl durchläuft zunächst ein $30 \mathrm{~cm}$ langes gegen Magnetfelder abgeschirmtes Rohr und beleuchtet einen am Ende dieses Rohres befindlichen $2-\mu$-Metallspalt. Der so ausgeblendete Strahl bewegt sich dann in einem $30 \mathrm{~cm}$ langen Messingrohr. Auf dieser Strecke greift das magnetische Erdfeld ein und lenkt die Elektronen je nach ihrer Geschwindigkeit ab. In $\mathrm{Abb} .5$ stellen wir neben dem Schattenbild des Spaltes einen sich nach links erstreckenden Schweif fest, der von Elektronen geringerer Geschwindigkeit gezeichnet ist. Zunächst

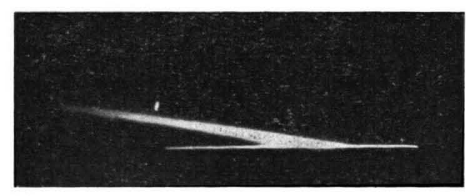

Ibb. 5. Geschwindigkeits-Analyse von Elektronenstrahlen aus der Gasentladungskathode durch Einwirkung des Magnetfeldes der Erde. ist man überrascht, daß das Spektrum so intensiv in Erscheinung tritt und geneigt, das oben zitierte Gerthsen-Fauldrahtsche Ergebnis anzuzweifeln. Aber eine im folgenden Kapitel beschriebene Wiederholung der Vergleichsmessung der Intensität des Schweifes zum Primärstrahl belehrt uns, daß die Hauptintensität bis auf einen verschwindend kleinen Bruchteil im Primärfleck liegt. Die Intensität des Primärflecks ( aralleler Elektronenstrahl) ist im vorher beschriebenen Experiment so groß, daß man nur schwer durch Verkürzung der Belichtungszeit Überbelichtung des Primärflecks vermeidet. Eine Verringerung der Elektronendichte läßt sich durch Einfügen eines elektrostatischen Objektivs unterhalb der Kathode leicht vornehmen. Es wird eine Bohrung von $0,1 \mathrm{~mm}$ im Durchmesser mit Elektronen aus der Gasentladung beleuchtet und mittels des Objektivs in der Ebene des $2-\mu$ Spaltes abgebildet. Dann entsteht ein Schattenbild des Spaltes mit dem durch das Erdmagnetfeld hervorgerufenen Spektrum. Hier zeigt sich nun ebenfalls, daß die Primärlinie stark überbelichtet sein muß, wenn das Spektrum erscheinen soll. Die Intensität

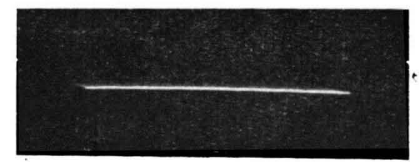

Abb. 6. Spektrum wie in Abb. 5, nur Intensität stark geschwächt und chromatischen Schweif durch Filterwirkung einer elektrischen Linse und durch Einsetzen einer Blende mit $0,05 \mathrm{~mm}$ Bohrung in den Brennpunkt des Objektivs abgeschnitten. 
des Schweifes ist also im Vergleich zum Primärfleck äußerst gering. Das Spektrum bricht im Gegensatz zu Abb. 5 schon nach etwa $1 \mathrm{~mm}$ ab. Der Grund dafür liegt in folgendem: Die elektrostatische Linse reflektiert alle Elektronen, deren Geschwindigkeit um mehr

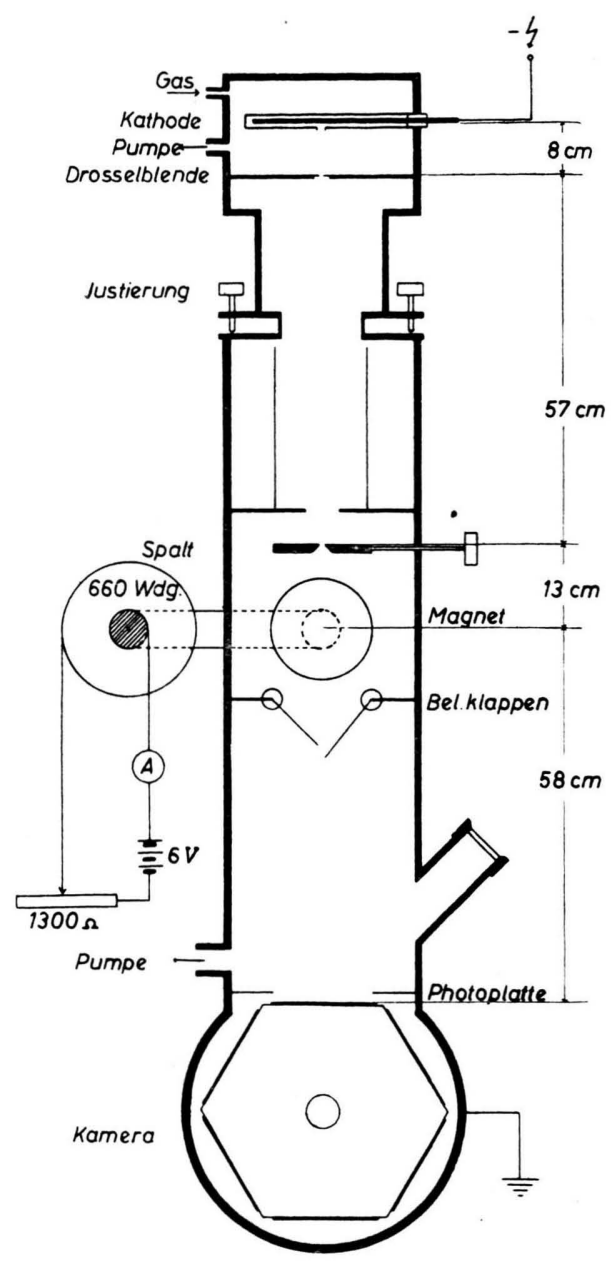

Abb. 7. Versuchsaufbau zur Geschwindigkeits-Analyse von Elektronen aus der Gasentladung mittels Magnetfelds. Auflösung: $100: 1$. als $10 \%$ geringer ist als die Geschwindigkeit, die sich aus der Kathodenspannung ergibt. Die Linse wirkt als Filter. Ferner kommt bei Vorhandensein einer Aperturblende in der Brennebene des Objektivs hinzu, daß Elektronen geringerer Geschwindigkeit nach dem Prinzip des Busch-Monochromators so stark verringert werden. Dies macht sich in Abb. 6 in der Weise bemerkbar, daß sich das durch das Magnetfeld der Erde hervorgerufene Spektrum bei starker Überbelichtung des Spaltbildes nur bis zu einer ganz bestimmten Grenze erstreckt.

Geschwindigkeits-Analyse im magnetischen Spektrographen

In Abb. 7 ist der benutzte Versuchsaufbau zur Bestimmung der spektralen Zusammensetzung der Elektronen aus der Gasentladung dargestellt. Als Kathode dient wieder die oben beschriebene Gasentladungskathode. Neben der Öffnung zum Einlassen des Gases ist die Öffnung zum Abpumpen des Gases eingezeichnet. Durch eine Drosselblende ist der Entladungsraum vom übrigen Rohr getrennt. Im Abstand von $57 \mathrm{~cm}$ befindet sich ein $4 \mu$ breiter Spalt, der mit Elektronen beleuchtet wird. Kurz darunter wird ein Magnetfeld erzeugt, dessen Kraftlinien senkrecht zur Flugrichtung der Elektronen stehen und zur GeschwindigkeitsAnalyse dienen. So ist das in Abb. 8 wiedergegebene Spektrum entstanden. Unten sind durch schrittweise Senkung der Kathodenspannung von $35 \mathrm{kV}$ auf 32,8 , $30,5,27,3,24,2,21,0$ und $17,1 \mathrm{kV}$ mittels der zwei Belichtungsklappen die Eichmarken für das Spektrum

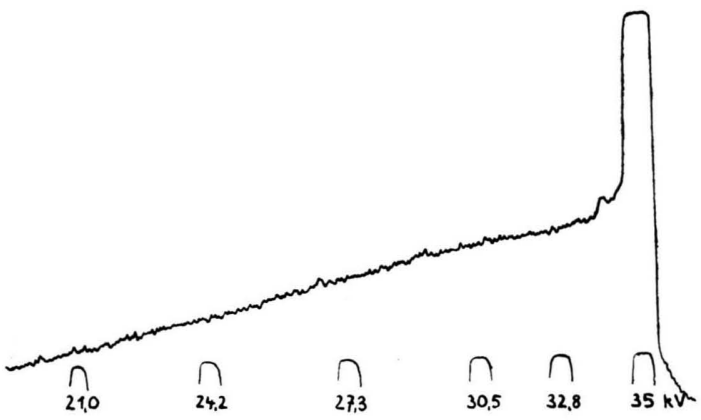

Abb. 9

Abb. 8. Mit dem magnetischen Spektrographen aufgenommenes Geschwindigkeitsspektrum von

Kathodenstrahlen aus der Gasentladung.

Abb. 9. Photometerkurve des in Abb. 8 wiedergegebenen Spektrums.

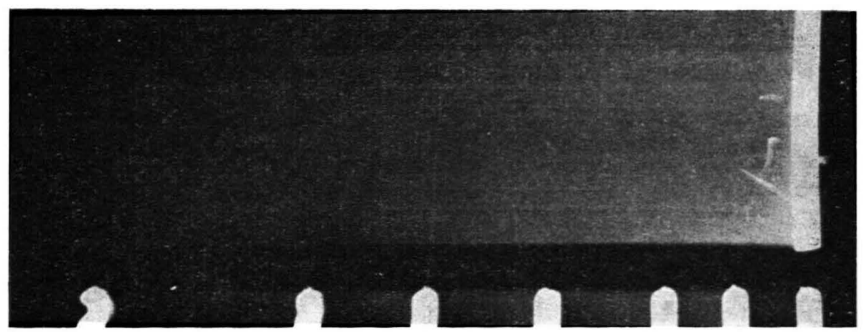

Abh. 8 
gedruckt. Das Spektrum besteht aus einer intensiven Linie mit einem sehr intensitätsarmen Schweif nach der Seite der verlangsamten Elektronen. Die Trennschärfe dieses einfachen magnetischen Analysators beträgt bei $35 \mathrm{kV}$ etwa $300 \mathrm{eV}$. Das bedeutet ein Auflösungsvermögen von $100: 1$. Die Photometerkurve des Spektrums Abb. 9 zeigt den kontinuierlichen Verlauf. Es zeigt sich keine Struktur, Stufe oder Linie. Die verlangsamten Elektronen stammen aus dem Gas. Die Intensität der Primärlinie ist infolge der starken Überbelichtung aus der Photometerkurve nicht angebbar. Um das Intensitätsverhältnis der Primärlinie zu der Gesamtintensität der langsameren Elektronen angeben zu können, wurde während der Aufnahme des Spektrums die Primärlinie abgedeckt (Abb. 10). Photometrisch konnte aber auch dadurch noch kein sicheres Ergebnis erzielt werden, da selbst bei einem Belichtungsverhältnis von 1:100 (Belichtungszeit der Primärlinie 0,5 sec, das übrige $50 \mathrm{sec}$ ) die Primärlinie immer noch überbelichtet war. Es konnte aber festgestellt werden, daß die Gesamtintensität der langsameren Elektronen unter $0,01 \%$ der Intensität der Primärlinie liegt. Diese Verhältnisse ändern sich auch nicht, wenn man als Entladungsgas Stickstoff oder Wasserstoff an Stelle von Luft benutzt. Auch eine Variation der Hochspannung von 17 bis $45 \mathrm{kV}$ ließ bei dieser geringen Auflösung des magnetischen Analysators keine Änderung im Spektrum erkennen.

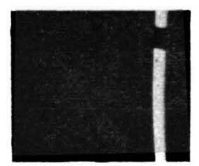

Abb. 10. Die Primärlinie wurde $0,5 \mathrm{sec}$ und die langsameren Elektronen 50 sec lang belichtet. Trotzdem ist die Primärlinie noch überbelichtet.

4. Hochauflösende Geschwindigkeits-Analyse im elektrostatischen Analysator

a) Analyse von Elektronen aus der InduniKathode

Wir untersuchen im folgenden mit dem hochauflösenden elektrostatischen Geschwindigkeits-Analysator $^{6}$ die chromatische Zusammensetzung der Elektronen, die in den bisherigen Spektren die intensive Primärlinie zeichneten. Der Versuchsaufbau ist in der schematischen Zeichnung, Abb. 11, dargestellt. Unterhalb der Gasentladungskathode befindet sich ein normales elektrostatisches Objektiv mit der üblichen Aperturblende von $0,05 \mathrm{~mm}$ im Durchmesser in der bildseitigen Brennebene. Da der im Vakuum verstellbare Feinspalt von 1-2 $\mu$ sich in einer Entfernung von $40 \mathrm{~cm}$ vom Objektiv befindet, wird er mit einem Elektronenbündel bestrahlt, dessen Apertur 0,05:400 $10^{-4}$ beträgt.

${ }^{6}$ G. Möllenstedt u. F. Heise, Physik. Bl. 5, 80 [1949]; G. Möllensted t, Optik 5, 499 [1949]; G. Möl lenstedt, Z.Naturforschg. 7 a, 465 [1952]: G. Mällenstedt. Optik 9, 473 [1952].
Damit ist die Voraussetzung für hohe Auflösung des Analysators gegeben. Das Potential für die nach dem schon in früheren Arbeiten beschriebenen Prinzip analysierende Zylinderlinse wird von einem 1200-MOhm-Potentiometer abgegriffen. Die Kathodenspannung kann in Sprüngen von 10 Volt gegenüber der Analysatorspannung erniedrigt werden, womit die Möglichkeit zum Drucken von Eichmarken gegeben ist. Ein Kondensator von $0,2 \mu \mathrm{F}$ dient zur Verringerung der Welligkeit der Hochspannung.

Ergebnisse: Stellt man den Analysator auf maximales Trennvermögen ein (Nachweis von $1 \mathrm{eV}$. Energieverlust bei $50000 \mathrm{eV}$ Strahlenergie) und läßt den durch den 2-»-Spalt ausgeblendeten Strahl durch die Randzone der Analysatorlinse laufen, so

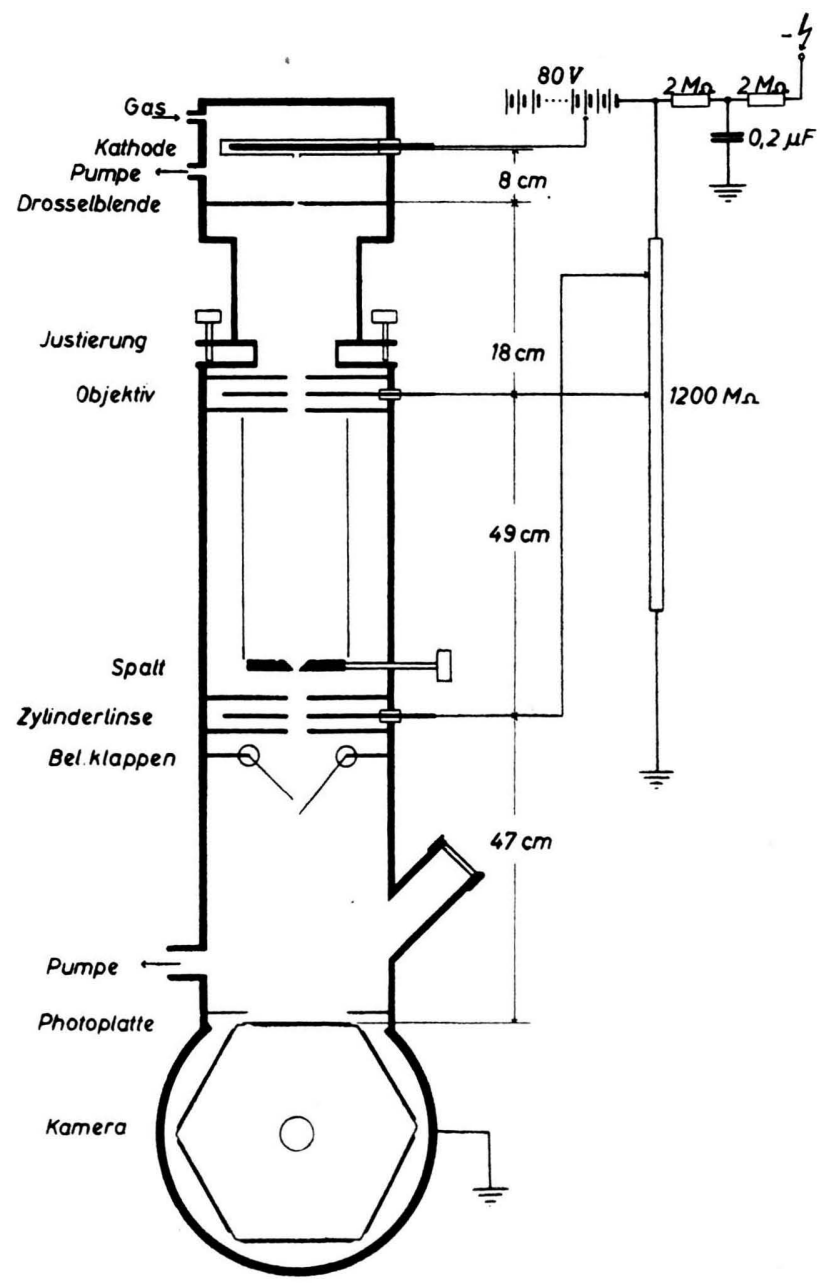

Abb. 11. Schaltung des hochauflösenden elektrostatischen Geschwindigkeits-Analysators mit Gasentladungskathode. 


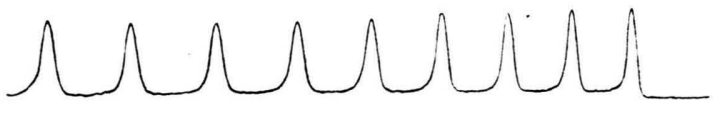

a)

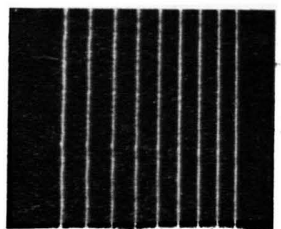

b)
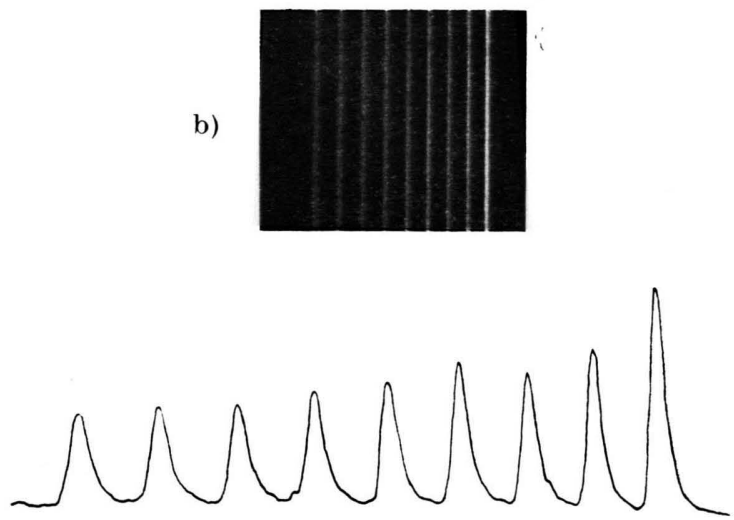

Abb. 12. a) Eichmarken mit Elektronen aus der Glühkathode. Strahlspannung: $35 \mathrm{kV}$. Kathodenspannungserniedrigung in acht Sprüngen um jeweils 10 Volt (Anodenbatterie).

b) Eichmarken mit Elektronen aus der Gasentladungskathode. Die langsameren Elektronen sind links im Spektrum registriert.

Oben: Photometerkurve von a. Unten: Photometerkurve von $b$.

ist man überrascht, welche scharfe Linie man auch mit Elektronen aus der Gasentladungskathode erhält. In der unteren Hälfte der Abb. 12 sind die Eichmarken der Elektronen aus der bei $40 \mathrm{kV}$ brennenden Gasentladung mit den Eichmarken, die mit Elektronen aus einer Glühkathode bei ebenfalls $40 \mathrm{kV}$ gedruckt wurden, zum Vergleich gegenübergestellt. Es zeigt sich, daß die unteren Linien fast die gleiche Schärfe wie die oberen haben. Erst bei starker Belichtung zeigen sich Abweichungen vom Spektrum der Glühelektronen. In Abb. 13 gewannen wir die Eichmarken durch Verringerung der Strahlspannung um jeweils 10 Volt durch Zuschalten von Spannungsstufen einer auf Hochspannung befindlichen Anodenbatterie. Die Spektren a, b, c und $\mathrm{d}$ wurden bei $50 \mu \mathrm{A}, 150 \mu \mathrm{A}, 200 \mu \mathrm{A}$ und $300 \mu \mathrm{A}$ Entladungsstrom aufgenommen. Links von der Hauptlinie sind die langsameren Elektronen registriert. Zunächst fällt die Asymmetrie der intensiv
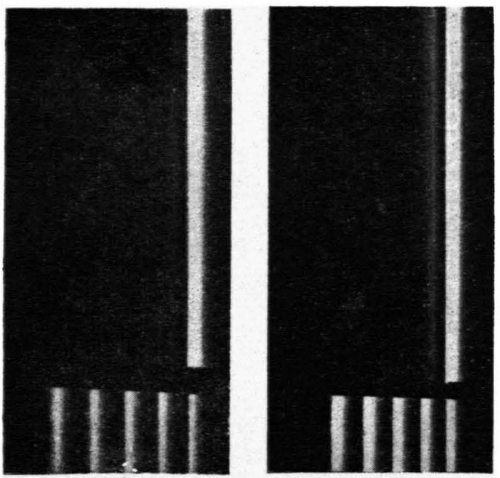

b)
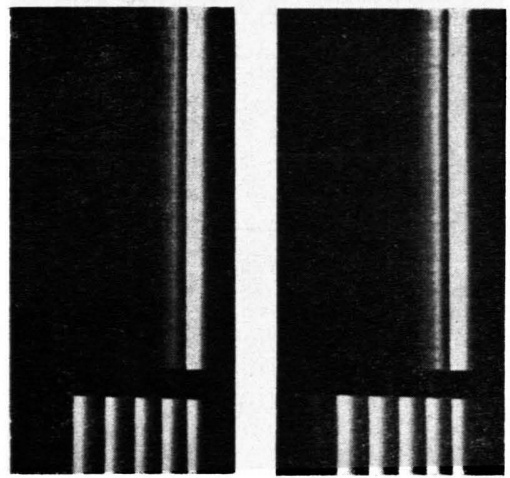

d)

Abb. 13. Stark belichtete Geschwindigkeitsspektren aus der Gasentladungskathode bei a) $50 \mu \mathrm{A}$, b) $150 \mu \mathrm{A}$, c) $200 \mu \mathrm{A}$ und d) $300 \mu \mathrm{A}$ Entladungsstrom. Die langsamen Elektronen befinden sich auf der linken Seite des Spektrums. Die Eichmarken wurden durch Erniedrigung der $40 \mathrm{kV}$ betragenden Kathodenspannung um jeweils 20 Volt erzielt.

gezeichneten Linie auf. Die steile Flanke befindet sich auf der Seite der langsameren Elektronen. Nach einer Intensitätslücke von $13 \mathrm{eV}$ zeichnet sich eine schwache aber scharfe Kante ab, an die sich ein kontinuierlicher Untergrund anschließt. Eine Photometerkurve der Abb. 13 läßt die Struktur des Spektrums deutlicher erkennen (Abb. 14).

In weiteren Experimenten wurde geprüft, ob die Struktur der Hauptlinie des Spektrums vom Kathodenmaterial oder vom Entladungsgas abhängig ist. Als Kathodenmaterial wurde Aluminium und Platin und als Gas Luft, reiner Stickstoff und Wasserstoff benutzt. Sämtliche Spektren wurden gleich lange und bei gleicher Temperatur des Ent. wicklers entwickelt. Um auch Intensitäten aus den Photometerkurven angeben zu können, wurden Schwärzungskurven durch Variation der Belichtungszeit aufgenommen und in derselben Weise entwickelt. Das Ergebnis der Messungen zeigt die in 


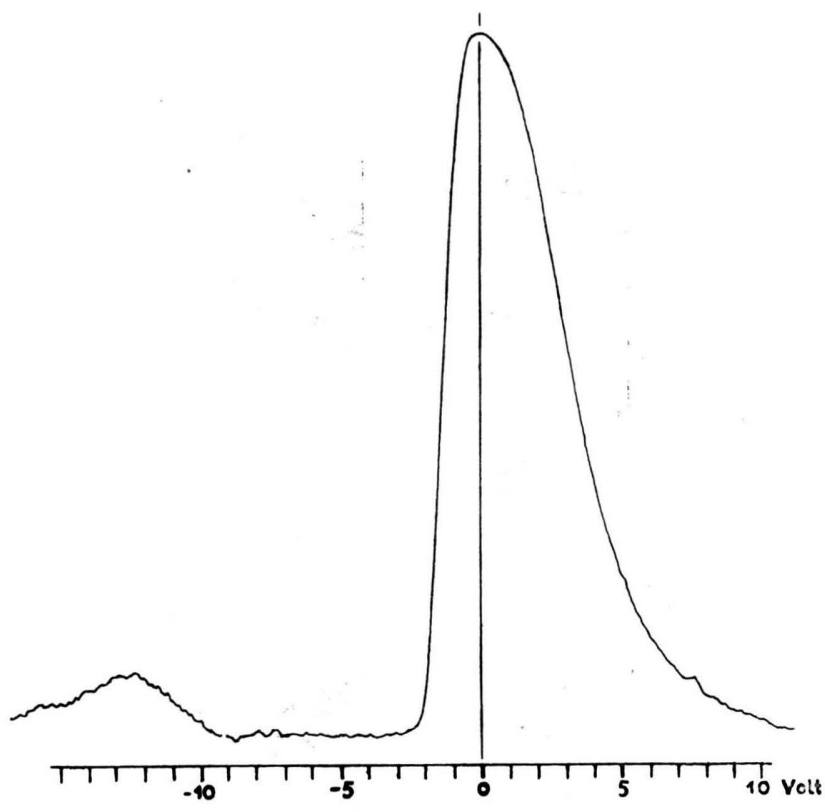

Abb. 14. Die Photometerkurve der Abb. 12 demonstriert deutlich die Unsymmetrie der Hauptlinie und den 13-eV-Energieverlust am Stickstoff-Molekül, das als Entladungsgas verwendet wurde.

100

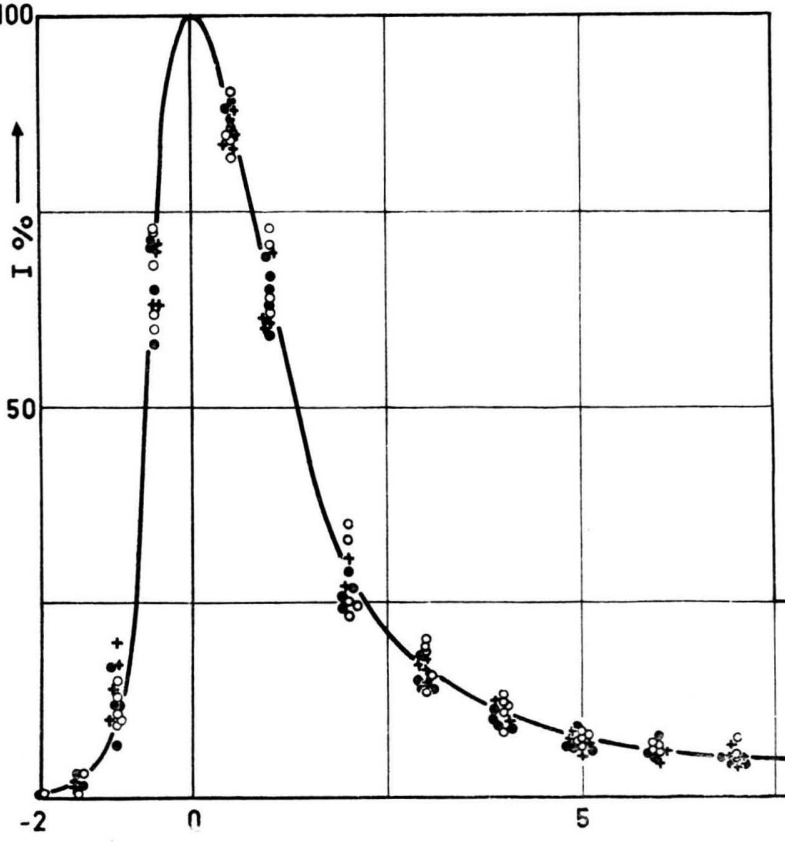

Abb. 15 wiedergegebene Kurve. Es demonstriert deutlich, daß die Form der asymmetrischen Kurve weder vom Kathodenmaterial noch von der Art des Entladungsgases abhängt.

b) Analyse von Elektronen aus einer Entladungsquelle mit durchbohrter Kathode nach Hailer

Im Gegensatz zu unseren bisherigen Versuchen verwendete Hailer ${ }^{7}$ eine Gasentladungsquelle, deren Kathode durchbohrt war, so daß nach Meinung Hailers die Elektronen nicht vom Kathodenmaterial ausgehen, sondern aus dem Gasraum stammen.

Es interessiert uns hier die chromatische $\mathrm{Zu}$ sammensetzung der aus dieser Kathode stammenden Elektronen. Die in Abb. 16 wiedergegebene Kathode wurde an Stelle der InduniKathode eingesetzt. Zwei zur Isolation dienende Glasrohre von $20 \mathrm{~cm}$ Länge und $4 \mathrm{~cm}$ Durchmesser wurden an den beiden Enden mit Metallflanschen versehen und in der in Abb. 16 wiedergegebenen Weise zusammengesetzt. Die Flansche dienen als Elektroden des Entladungssystems. Um ein ruhiges Brennen der Entladung zu erzielen, wurde das untere Rohr mit einer dünnen Platinschicht bedampft, deren Dicke von unten nach oben zunimmt. So stellt sich eine gleichmäßige Potentialverteilung ein. Die Mittelelektrode dient als Kathode. Die oberste Elektrode wurde an ein Zwischenpotential gelegt, damit die durch den Kanal der Mittelelektrode fliegenden Ionen abgebremst werden. In die Anode ist zur Erzeugung eines feinen Strahls eine 50- $\mu$-Blende eingesetzt. Um möglichst stabile Verhältnisse zu haben, wurde ein Messingröhrchen von $40 \mathrm{~mm}$ Länge und $3 \mathrm{~mm}$ Durchmesser als Kanal in die negative Elektrode eingesetzt. Der Entladungs-

Abb. 15. Unabhängigkeit des Energiespektrums vom Entladungsgas (Luft, Wasserstoff, Stickstoff) und vom Kathodenmaterial (Aluminium und Platin). Kathodenspannung etwa $40 \mathrm{kV}$.

${ }^{7}$ C. Hailer, Wiss. Veröff. Siemens-Werken 17, 115 [1938]. 


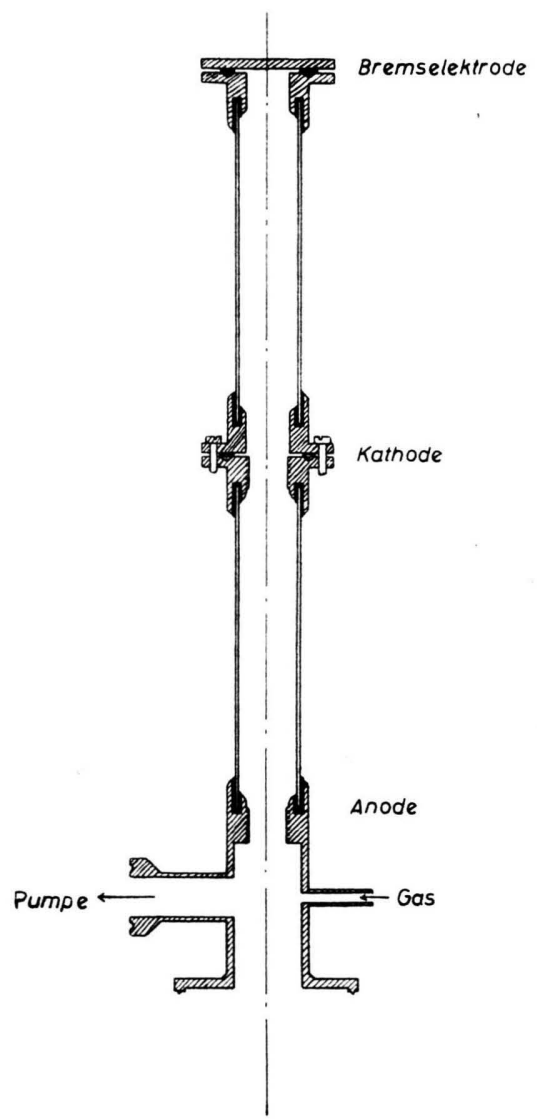

Abb. 16. Gasentladungskathode mit durchbohrter Elektrode (,nicht materielle“" Kathode).

pinsel setzte zunächst nicht im Kanal an, sondern sprang an der mit Platin bedampften Glasröhre hin und her. Erst nach Bedampfen des Messingröhrchens mit Platin führte der Entladungspinsel in den Kanal.

Um sicher zu gehen, daß die in den Spalt des Geschwindigkeits-Analysators fallenden Elektronen auch aus der Mitte des Kanals stammten, wurde mittels Lochkamera (Abb. 17) die Kanalöffnung abgebildet, indem man zunächst Spalt und Analysator aus der Apparatur entfernte. In der Abb. 18 erscheint der Kanalrand als Ring. Die aus der Mitte des Kanals kommenden Elektronen zeichnen den Mittelfleck. Belegt man die Innenwand des Messingröhrchens mit Platin, so ergibt sich Abb. 19. (Im Gegensatz zu Abb. 18 ist der Mittelfleck wesentlich intensiver.) Beide Aufnahmen wurden unter sonst gleichen Bedingungen und mit der gleichen Belichtungszeit aufgenommen. Dieses läßt vermu-

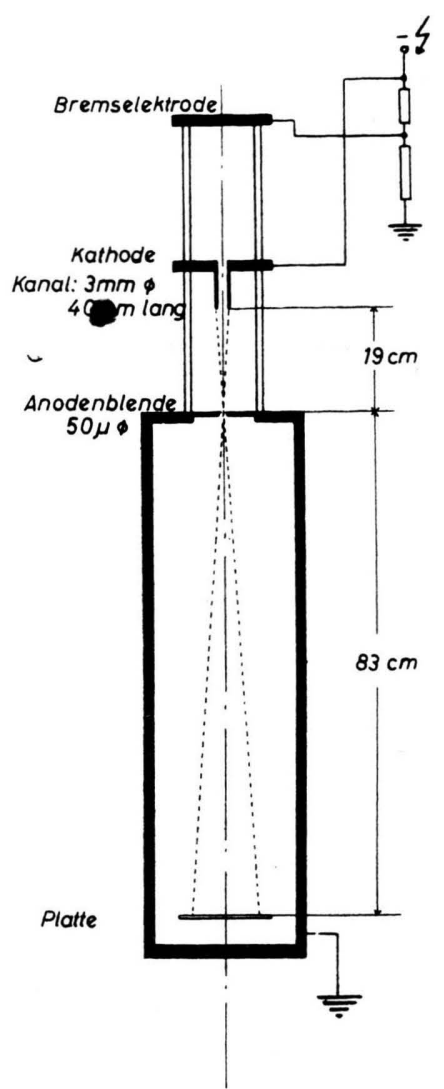

Abb. 17. Versuchsanordnung zur Lochkamera-Abbildung der Elektronenquelle bei einer Gasentladungskathode mit durchbohrter Elektrode.

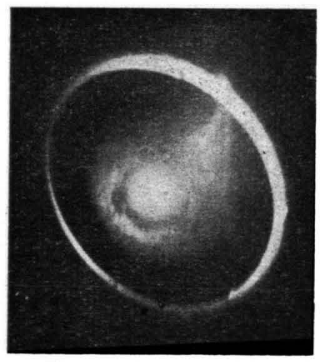

Abb. 18. Lochkamerabild der Elektronenquelle bei eingesetztem Messingkanal.

ten, daß bei der durchbohrten Kathode die Innenwand des Kanals wesentlich an der Elektronenlieferung beteiligt ist.

Benutzt man für die Analyse mit dem elektrostatischen Analysator den in Abb. 18 und 19 wiedergegebenen Mittelfleck, so erhält man das in Abb. 20 gezeigte Spektrum. Es besteht aus einer relativ 


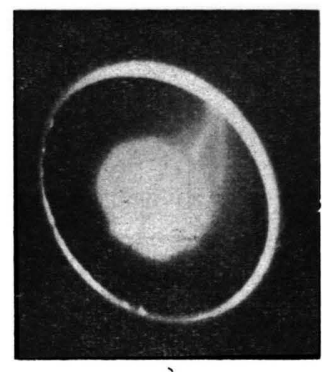

Abb. 19. Lochkamerabild der Ektrone quelle bei eingesetztem platinierten Kanal.

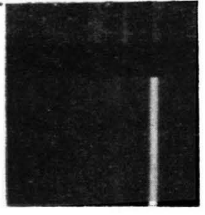

Abb 20. Spektrum der Elektronen aus einer Gasentladung mit durchbohrter Kathode.

scharfen asymmetrischen Linie, die vcllkommen dem Spektrum von Elektronen gleicht, die aus einer Gasentladung mit ,,materieller" Kathode stammen (s. Photometerkurve Abb. 21). Auch eine Erweiterung des Kanals auf $24 \mathrm{~mm}$ bringt keine Änderung der spektralen Verteilung.

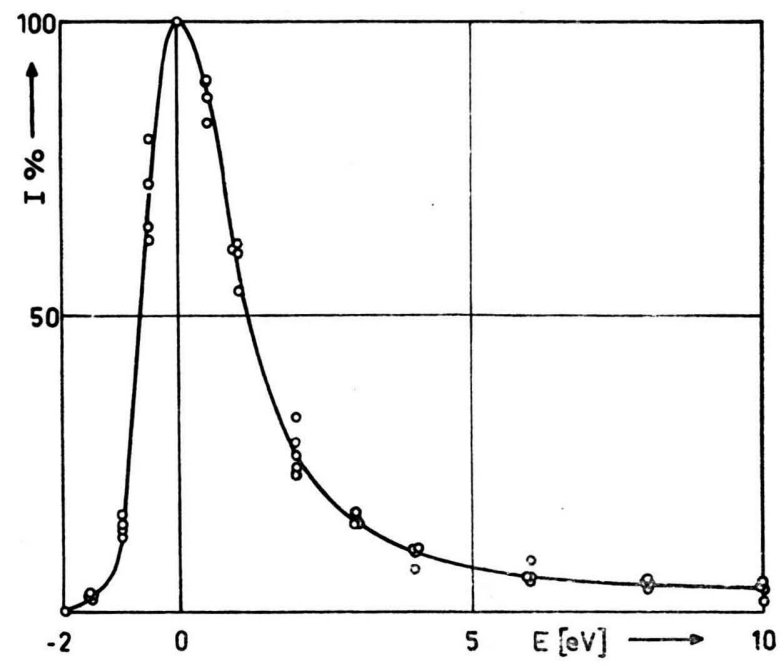

Abb. 21. Photometerkurve der Hauptlinie des in Abb. 20 wiedergegebenen Spektrums.

Nach diesen Versuchen ist man geneigt anzunehmen, daß nicht alle Ionen, die in den Kanal eintreten, diesen auch am Ende wieder verlassen. Ein großer Teil wird auf der Kanalwand landen und dort Elektronen auslösen. Für diese Auffassung spricht auch die Beobachtung, daß die Innenwand des Kanals nach längerer Betriebsdauer mit einer
Kohleschicht belegt ist, die sich an Metallflächen infolge vorhandener Öldämpfe und Fettmoleküle an Auftreffstellen von Ionen bildet.

c) Sekundär-Elektronen aus einer im Hochvakuum befindlichen Kathode

Bei den oben beschriebenen Versuchen tritt ein Teil der zu analysierenden Elektronen auf dem Wege von der Kathode bis zur Anode mit den Atomen bzw. Molekülen des unter einem Druck von $10^{-2} \mathrm{~mm} \mathrm{Hg}$ stehenden Entladungsgases in Wechselwirkung. Um dieses zu vermeiden, wurde eine Apparatur gebaut, bei der die Ionen in einem besonderen Entladungsrohr erzeugt werden. Der Kanalstrahl wird unter $30^{\circ}$ auf eine in einem Vakuum von $10^{-4} \mathrm{~mm} \mathrm{Hg}$ befindliche Kathode geschossen. Die so ausgelösten Elektronen laufen also nur durch Hochvakuum. Man erhält auf diese Weise ein reines Spektrum der durch Ionenbeschuß ausgelösten Elektronen. Abb. 22 zeigt das mit dem elektrosta-

Abb. 22. Spektrum von Elektronen, die durch Ionen ausgelöst wurden, die unter $30^{\circ}$ aus einem getrennten Entladungsraum auf die in einem Vakuum von $10^{-4} \mathrm{~mm} \mathrm{Hg}$ befindliche Kathode geschossen wurden. Eichmarken : 20 eVolt.
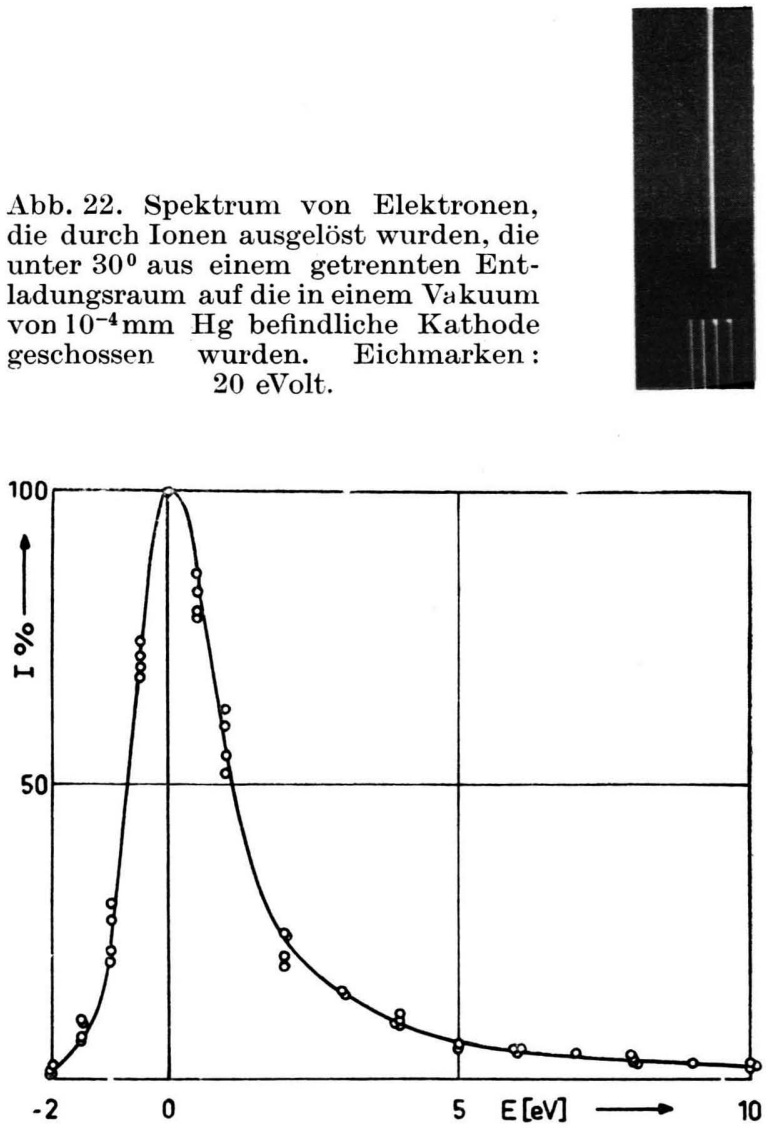

Abb. 23. Durch Auswertung der Aufnahme 22 erhaltene Geschwindigkeitsverteilung. 
tischen Analysator aufgenommene Geschwindigkeitsspektrum. Als Kathodenmaterial diente V2A. Stahl und als Entladungsgas Luft. Die Geschwindigkeitsverteilung der auf diese Weise ausgelösten Elektronen ist durch Auswertung von Photometerkurven in Abb. 23 wiedergegeben. Die spektrale Verteilung unterscheidet sich nicht von den bisher gefundenen. Selbstverständlich fehlt in dem Spektrum die 13-eV-Verlustkante, da die ausgelösten Elektronen keine Verluste am Entladungsgas erleben.

\section{Endergebnis}

In Abb. 24 sind die in den vorher beschriebenen Experimenten erhaltenen Ergebnisse zusammengefaßt. Man erkennt, daß trotz der verschiedenen Ver-
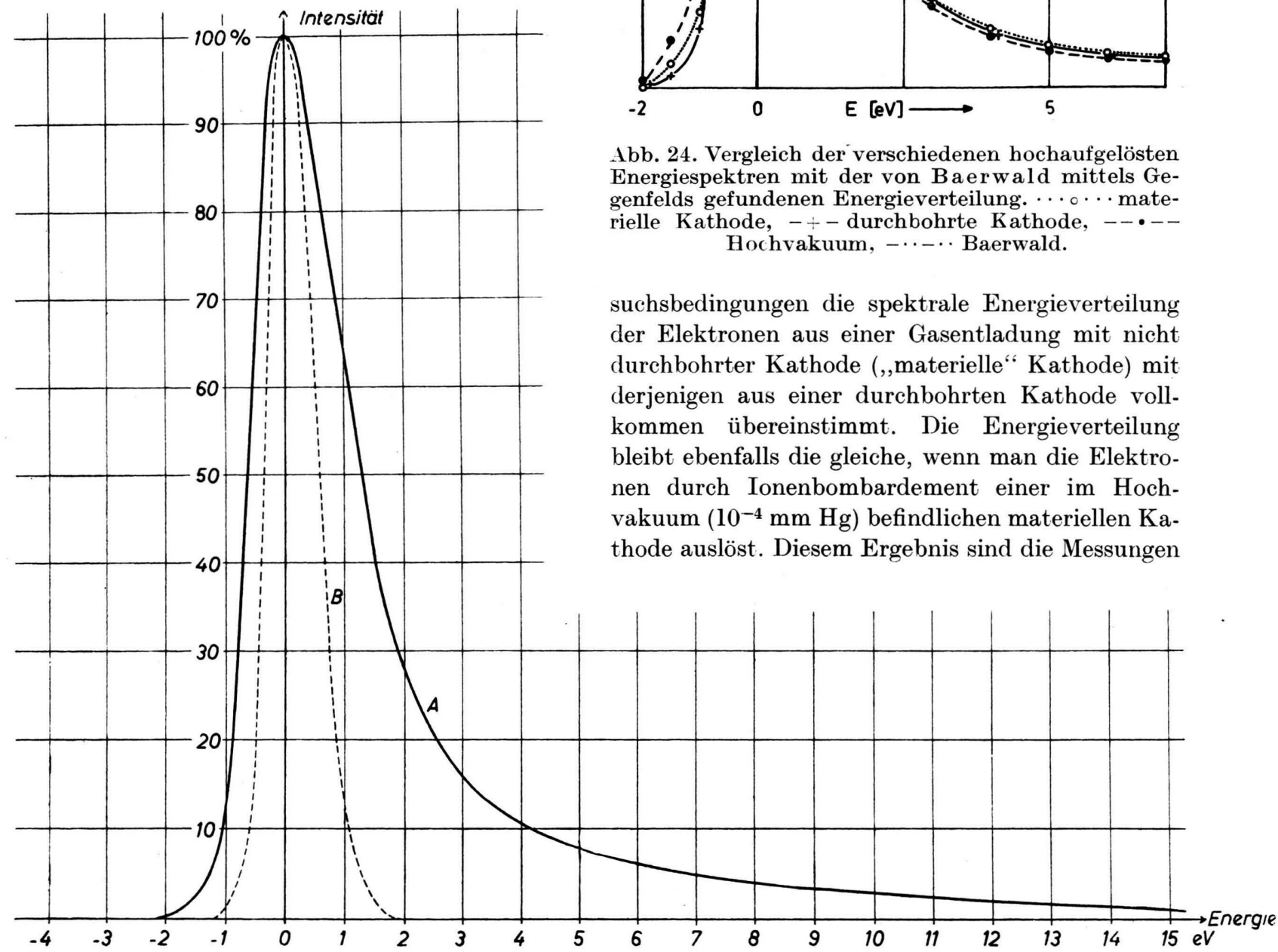

Abb. 25. Mit dem elektrostatischen Analysator bestimmte spektrale Energieverteilung

A) von 40-keV-Elektronen, die durch 40-keV-Ionen ausgelöst wurden,

B) von $40-\mathrm{keV}$-Elektronen, die aus einem bei $2900^{\circ} \mathrm{C}$ glühenden Wolframdraht austreten. Linie verbreitert durch begrenztes Auflösungsvermögen des Analysators.

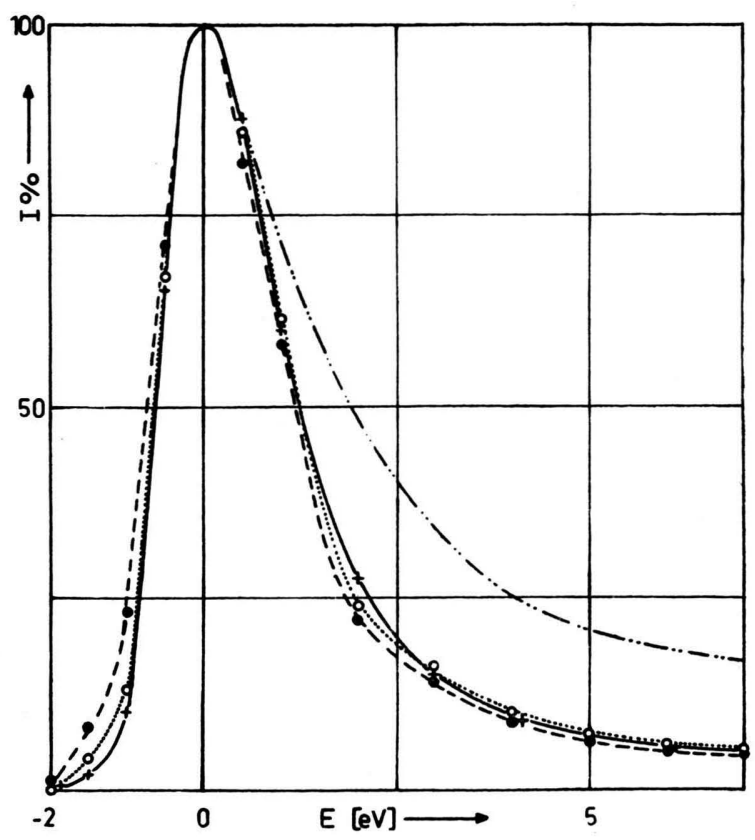

Abb. 24. Vergleich der verschiedenen hochaufgelösten Energiespektren mit der von Baerwald mittels Gegenfelds gefundenen Energieverteilung. $\cdots \circ \cdots$ materielle Kathode, - +-durchbohrte Kathode, --•-Hochvakuum, - - - B Baerwald.

suchsbedingungen die spektrale Energieverteilung der Elektronen aus einer Gasentladung mit nicht durchbohrter Kathode (,,materielle" Kathode) mit derjenigen aus einer durchbohrten Kathode vollkommen übereinstimmt. Die Energieverteilung bleibt ebenfalls die gleiche, wenn man die Elektronen durch Ionenbombardement einer im Hochvakuum $\left(10^{-4} \mathrm{~mm} \mathrm{Hg}\right)$ befindlichen materiellen $\mathrm{Ka}$ thode auslöst. Diesem Ergebnis sind die Messungen 
von Baerwald ${ }^{8}$ gegenübergestellt. Er hatte mittels der Gegenfeldmethode die Geschwindigkeitsverteilung der durch 30-kV-Ionen ausgelösten SekundärElektronen gemessen. Die Energieverteilung ist etwas breiter als die mit dem elektrostatischen Analysator gemessene. Die Ursache hierfür kann in der Verwendung eines nicht genügend feinmaschigen Netzes für die Gegenfeldmethode liegen. In Abb. 25 ist die spektrale Energieverteilung der Gasentladungs-Elektronen mit dem Spektrum der Glühelektronen (Wolfram $2900^{\circ} \mathrm{C}$ ) verglichen. Der Null-

${ }^{8}$ H. Baerwald, Ann. Physik 41, 643 [1913]. wert der Abszisse entspricht nicht der Energie 0 eVolt, sondern die Spektren sind so verschoben, daß ihre Maxima zusammenfallen.

Die Verfasser vermuten, daß die charakteristische Asymmetrie der Energieverteilung der Elektronen in der nicht einheitlichen Geschwindigkeit der aufprallenden Ionen liegt. Diese Frage soll später durch Energiespektren von Elektronen, die durch Ionen einheitlicher Geschwindigkeit ausgelöst wurden, entschieden werden.

Unser ganz besonderer Dank gilt Herrn Professor Dr. W. Kossel für sein reges Interesse an diesen Untersuchungen.

\title{
Bestimmung der photoelastischen Konstanten $p$ und $q$ optischer Gläser
}

\author{
Von Clemens Schaefer und Heinrich Nassenstein \\ Aus dem II. physikalischen Institut der Universität Köln \\ (Z. Naturforschg. 8a, 90-96 [1953]; eingegangen am 27. November 1952)
}

Herrn Prof̣essor Erwin Fues zum 60. Geburtstag gewidmet

\begin{abstract}
Während in den letzten Jahren die photoelastischen Konstanten einer größeren Zahl von Stoffen ermittelt wurden, fehlt es bisher an einer zusammenfassenden Untersuchung: der optischen Gläser. Es liegen nur Einzelbeobachtungen vor, die aber auch zum größten Teil nur historisches Interesse beanspruchen, da die in die Rechnung eingehenden elastischen Konstanten nicht ermittelt waren, oder aber die Glassorten selbst nicht bekannt sind oder nicht mehr hergestellt werden. Es wurden daher im II. Physikalischen Institut der Universität Köln von 154 verschiedenen optischen Gläsern der Firma Schott \& Gen. die photoelastischen Konstanten bestimmt, deren Werte in dieser Arbeit mitgeteilt werden.
\end{abstract}

\section{Das Meßverfahren}

$\mathrm{D}$ ie Messung der photoelastischen Konstanten erfolgte in Anlehnung an eine Arbeit von Pok $\mathrm{kels}^{1}$ nach folgendem Verfahren: Von jeder Glassorte schnitt man zwei annähernd gleiche Quader (etwa $9 \cdot 19 \cdot 19 \mathrm{~mm}^{3}$ ). Diese wurden in die beiden Strahlen eines Jaminschen Interferentialrefraktors gebracht, und der eine senkrecht zur Fortpflanzungsrichtung des Strahles komprimiert. Die hierbei auftretende Verzögerung des ersten Strahles im komprimierten Glase wurde durch die Streifenverschiebung im Fernrohr des Jamin gemessen. Da die Strahlen durch die ungefähr unter $55^{0}$ erfolgende Reflexion an den Spiegeln des Jamin schon weitgehend linear polarisiert waren, erübrigte sich die Verwendung eines Polarisators.

Für die relative Streifenverschiebung $d$ (d. h.

${ }^{1}$ F. Pockels, Ann. Physik 7, $745[1902]$.
Streifenverschiebung $\partial$ dividiert durch Streifenabstand $d$ ) ergeben sich dann die Formeln ${ }^{2}$ :

a) bei Druck senkrecht zur Polarisationsebene (Abb. 1):

$$
\Delta_{z}=\left(\frac{\delta}{d}\right)_{z}=\frac{n P_{1}}{\lambda B E}\left\{v\left(1-\frac{1}{n}\right)+\frac{q}{\omega}-2 v \frac{p}{\omega}\right\},
$$

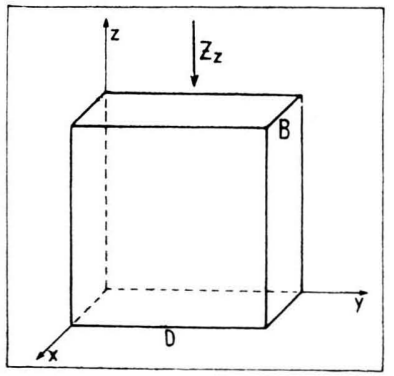

Abb. 1.

$$
{ }^{2} \text { s. F. Pockels, l. c. }{ }^{1} \text {. }
$$

